\title{
Prediction of Channel Availability in Cognitive Radio Networks Using a Logistic Regression Algorithm
}

\author{
Hans Marquez ${ }^{\# * 1}$, Cesar Hernández ${ }^{\# 2}$, Diego Giral ${ }^{\# 3}$ \\ \#Universidad Distrital Francisco José de Caldas, Bogotá-Colombia. \\ ${ }^{*}$ Colciencias, Bogotá-Colombia. \\ 1'hans.marquez.ramos@gmail.com; ${ }^{1}$ cahernandezs@udistrital.edu.co; \\ 3agiralr@correo.udistrital.edu.co
}

\begin{abstract}
The capacity of predicting spectral occupancy in cognitive radio networks offers the possibility of developing better policies in channel assignment to secondary users, according to the predicted spectral opportunities. This work develops a prediction model to determine and exploit spectral opportunities while avoiding the continuous search for channel availability in cognitive radio networks. The proposed scheme creates an availability prediction matrix for every available channel in the GSM band that includes their times of availability. By using this information, there is a potential to improve channel allocation policies. The model contains two processes: the first one performs a training process in order to prepare the prediction algorithm so that it can make more reliable predictions and the second one uses the logistic regression algorithm to estimate the availability in every available frequency which can be profited by secondary users, who intend to start transmissions. Measurements were made for average bandwidth, average delay and prediction error. The results obtained were evaluated with real spectral occupancy data in the GSM frequency band. The developed model shows a low prediction error which enables optimal channel assignment mechanisms, hence minimizing failed handoffs through the channel occupation of primary users.
\end{abstract}

Keyword - Availability, Cognitive radio, Logistic regression, Prediction.

\section{INTRODUCTION}

Cognitive radio is defined as a "radio system that knows its environment and can dynamically and autonomously adjust its radio operation parameters" [1]-[3] and offers a solution to the current problem that communications face which is the scacrcity in the available spectrum. However, there is a low use of the spectrum in some bands while some bands are completely saturated. To deal with this issue, the study on cognitive radio network (CRN) began whose advantages would allow a more flexible use of the spectrum therefore optimizing the already limited resources in wireless networks. This would not only avoid the rigidness in the current assignment of the spectrum but would also improve the quality of the service offered resulting in higher spectral efficiency.

The generated spectral opportunities allow a non-licensed user, a cognitive radio user or a secondary user (SU) to use a channel from an available licensed band until the primary user (PU) takes over that channel. Other cases include a decrease in the quality of the channel taken by the SU, the interference from a SU with the PcU's activity and the mobility of the SU leads him to an area out of coverage. Under these considerations, the SU should release this channel and search for a new one which is known a spectral handoff [4]-[6].

The method to avoid or minimize the interference on a PU is to force the SU to perform a handoff or change of channel before the presence/arrival of the PU is detected. This reduces the degradation of the channel quality so that the transmissions of the PU are not affected by the opportunistic use of the spectral resources on behalf of the SU. To generate these handoffs before the PU's arrival, a model is proposed to predict the spectral occupancy by estimating the possibility of a PU's arrival which would optimize the channel assignment process.

The present work is structured as follows: section 2 presents the related work, section 3 describes the development of the model, section 4 shows the results, and section 5 finishes with a set of conclusions.

\section{RELATED WORK}

In [7], the research focuses on how the increasing use of services inside a vehicle (cellphones, GPS and radio signals) has become a concern in terms of security. On that matter, the authors propose a system that detects the driver's distraction and adapts the vehicle to mitigate such distractions. To develop this system, the use of Support Vector Machines (SVM) was proposed to develop a real time approach for the cognitive distraction using the driver's ocular movements. The data was collected from a simulation with ten participants that interacted with the system while driving. These data were used to train the SVM and logistic regression models with the purpose of investigating three different characteristics: how was created the distraction, which data 
entered the model, and how was the input data summarized. The results show that the SVM models were capable of detecting the distraction with an average accuracy of $81.1 \%$ surpassing the logistic regression model.

In [8], a two-stage cognitive process is proposed with the purpose of learning from the capacities of the physical layer under different channel conditions which leads to an optimization of the package delivery in a multi-antenna radio. In the first step, it learns the characteristics of the available techniques and in the second step the configuration is defined according to the radio's goals and channel conditions. A design is proposed based on the Bayes rule which will be used as a baseline for future comparisons. In this work, the Naïve Bayes, Semi-Naïve Bayes and binary search models are studied since they offer several learning techniques and optimize the design used as a reference. It requires the estimation of fewer parameters but sacrifices optimization in terms of performance to save speed and memory.

In [9] the authors mention the main goals of cognitive radio (CR) pointing out that one of them consists on improving the inefficient use of the spectrum. It also states how cognitive radio can dynamically perceive the spectrum to gather information. Such gathering can be used to describe spectral opportunities as well as determining the future occupancy of the spectrum. In the work developed by the authors, binary time series are used to characterize and predict the occupancy of the spectrum. The deterministic and non-deterministic occupancy data is then examined showing the results over both scenarios.

In [10] the self-regressive linear approach for binary time series is adopted to study the performance in the prediction of the channel occupation based on the spectrum measurements carried out in a synchronized fashion in four different places. Through the modeling, the dependence of the adjacent frequencies in the frequency domain is factored. The order of the model is selected in terms of the measured residual magnitudes and the Akaike information criteria, by tabulating the results and considering the time of observation for each location. The performance of the proposed linear system is compared with the Markov chain model in continuous time for one of the locations.

In the research carried out by [11] the objective is to improve the detection probability using the regression of binary decision-making obtained from individual cognitive radio nodes between the primary user and the cooperative center of spectrum detection. It is an innovative technique that adds upgrades to the probability system using the deterministic nature of propagation loss from long distance radio in a distributed detection center. The simple model of Log-distance path loss is considered in this work and the Log-normal shading effect is assumed. The established framework can be extended to other variations and combinations of path loss models. It can also implement an advanced pattern of adaptation techniques.

In [12] the importance of the efficient use of the spectrum is pointed out due to the omnipresence of wireless technologies that are currently at disposal. The detection of the spectrum is a key stage towards an efficient use of the spectrum. The detection of energy is a fast and simple method to detect the spectrum but its accuracy is limited by the dependence on a threshold value. This article describes a new detection method for spectrum energy in real time using the logistic regression classifier. The implementation is performed using USRP and GNU-Radio, and reaches $98.6 \%$ accuracy in classification with a dataset collected over the commercial FM band.

\section{Methodology}

Fig. 1 shows the block diagram of the proposed prediction model. The first block called "Spectrum occupancy database" includes real occupancy data corresponding to the GSM band ( $824 \mathrm{MHz}-874 \mathrm{MHz}$ ). This block is the input for the spectrum data processing that has the task of defining the occupancy or availability of each channel in the GSM band according to the false alarm probability equation.

The rectangular area corresponds to the proposed model that consists of two algorithms (1) Logistic regression algorithm and (2) Channel assignment prediction. The first method's function is to train the algorithm for a ten-minute period through the use of training variables such as PSINR, availability and average availability time. This leads to the calculation of parameters known as cost and gradient which are necessary to adjust the predictor. The second method assigns the channel occupancy by setting " 1 " and "0" which outputs an availability prediction matrix regarding the bandwidth. 


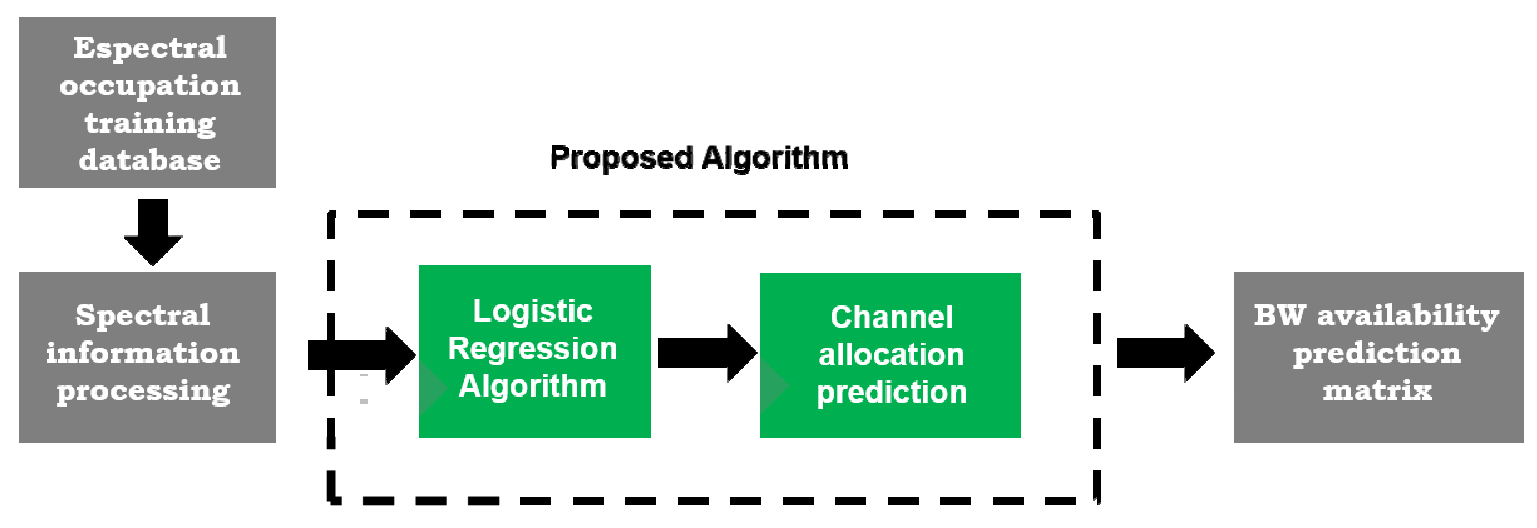

Fig. 1. General Structure of the Proposed Model.

\section{A. Defining simulation time and spectral occupancy data}

The model assigns the resources according to the existing demand taking into account the existing time intervals for simulation. It is noteworthy to mention that each time interval lasts $300 \mathrm{~ms}$ and the simulation time lasts 10 minutes.

Additionally, the model has a matrix with spectral occupancy data as inputs for the GSM frequency bands which significantly differentiate in terms of the traffic measured over different time intervals. Each differentiation refers to the volume of information and occupancy shown in each channel which generates high and low traffic data. This differentiation tests the model over two separate scenarios with their own characteristics.

\section{B. Logistic regression algorithm}

The main advantage of logic regression is that it can use diverse explicative variables at the same time. Although it may seem trivial, this characteristic is important due to the great interest on knowing the impact of these variables over the response variable. If the explicative variables were examined independently, ignoring the covariance between variables could lead to confusion.

One logistic regression will model the probability of the result based on individual characteristics and is given by the following equation:

$$
\log \left(\frac{\pi}{1-\pi}\right)=\beta_{0}+\beta_{1} x_{1}+\beta_{2} x_{2}+\cdots \beta_{m} x_{m}
$$

Where $\pi$ indicates the probability of an event, $\beta$ are the regression coefficients associated with the reference group and the xi explicative variables. At this point, an important concept has to be highlighted. The reference group represented by $\beta 0$ is constituted by those individuals that present a reference level for each variable $\mathrm{x} 1$, $\mathrm{x} 2, \ldots, \mathrm{m}$.

For the specific case of the present research, the following were defined as explicative variables: the signalto-interference-plus-noise ratio (SINR), the availability and the average availability time since they are all related and their simultaneous use is required in the prediction of the channel availability. Therefore, equation (1) would turn into equation (2):

\section{Model design}

$$
\log \left(\frac{\pi}{1-\pi}\right)=\beta_{0}+\beta_{1} \mathrm{PD}_{1}+\beta_{2} \text { TED }+\beta_{3} \text { PSINR }
$$

The proposed model takes as an input variable the spectral occupancy's training matrix. Before being used in the predictor's training process, the spectral information passes through the spectral information processing block which converts the data into dichotomy series where " 0 " represents the channel's occupation and "1" represents the channel's availability. With this information-processing phase the Logistic Regression algorithm is trained.

The purpose of the algorithm consists on using this dichotomy training matrix to implement a function $\mathrm{h}: \mathrm{x} \rightarrow$ $y$ where $h(x)$ is an effective predictor regarding the values of $y$ (see Fig. 2). 


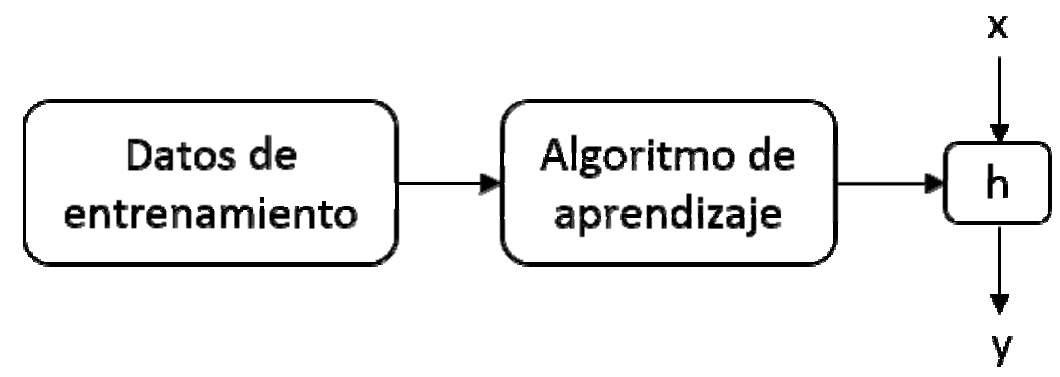

Fig. 2. Training Process of the Algorithm

According to equation (2), a hypothesis function for channel availability is created and is defined as shown in equation (3):

$$
\mathrm{h}_{\theta}(\mathrm{x})=\theta_{0}+\theta_{1} \mathrm{PD}_{1}+\theta_{2} \text { TED }+\theta_{3} \text { PSINR }
$$

Where $\theta_{\mathrm{i}} \mathrm{s}$ are parameters or weights (see equation (4)):

$$
\mathrm{h}(\mathrm{x})=\sum_{\mathrm{i}=0}^{\mathrm{n}} \theta_{\mathrm{i}} \mathrm{x}_{\mathrm{i}}=\theta^{\mathrm{T}} \mathrm{x}
$$

To learn or find the $\theta_{\text {values, }} \mathrm{h}(\mathrm{x})$ must be similar to $\mathrm{y}$ or minimize the cost function in equation (5):

$$
\mathrm{J}(\theta)=\frac{1}{2} \sum_{\mathrm{i}=0}^{\mathrm{m}}\left(\mathrm{h}_{\theta}\left(\mathrm{x}^{(\mathrm{i})}\right)-\mathrm{y}^{(\mathrm{i})}\right)^{2}
$$

By finding the values of $\theta$, all the necessary values of the hypothesis function can be defined and the algortithm can take them to perform predictions on spectral occupancy.

\section{IV.RESULTS}

In the present simulation, the prediction algorithm was tested under two scenarios: high traffic and low traffic. The metrics associated with the efficiency analysis of the present algorithm are classified into 8 metrics which are:

TABLE I. Simulation Metrics

\begin{tabular}{|c|c|c|c|}
\hline Handoffs & Bandwidth & Average delay & Throughput \\
\hline $\begin{array}{l}\text { Anticipated Perfect Failed } \\
\text { Successful Interference }\end{array}$ & $\begin{array}{c}\text { Cumulative average } \\
\text { bandwidth }\end{array}$ & $\begin{array}{c}\text { Cumulative average } \\
\text { delay }\end{array}$ & $\begin{array}{c}\text { Cumulative average } \\
\text { throughput }\end{array}$ \\
\hline
\end{tabular}

Fig. 3 to 10 show the results obtained for each metric previously mentioned both for high traffic and low traffic.

According to the results obtained both for high and low traffic, the number of anticipated handoffs is zero which indicates that the algorithm took advantage of the useful transmission time in the channels and abandoned the channel prior to the arrival of the primary user. This is reflected in the throughput level being relatively constant during the entire transmission as the bandwidth is (See Fig. 3).

It is seen that the number of cumulative handoffs in high traffic is higher than in low traffic. However, the number of handoffs with interference is lower in the low traffic scenario which indicates that the algorithm behaves better in scenarios with high traffic leading to a higher number of perfect handoffs (See Fig. 4 to 6).

In the case of failed handoffs (see Fig. 7), a particular behavior is seen in high traffic in contrast to the one shown in low traffic. The number of failed handoffs is significantly lower, indicating a better behavior of the algorithm in this type of scenario.

Regarding the delay, it is evidenced that the cumulative delay is higher in high traffic. This result is consistent due to the number of cumulative handoffs during simulation which is lower than the one registered for low traffic (see Fig. 8). 


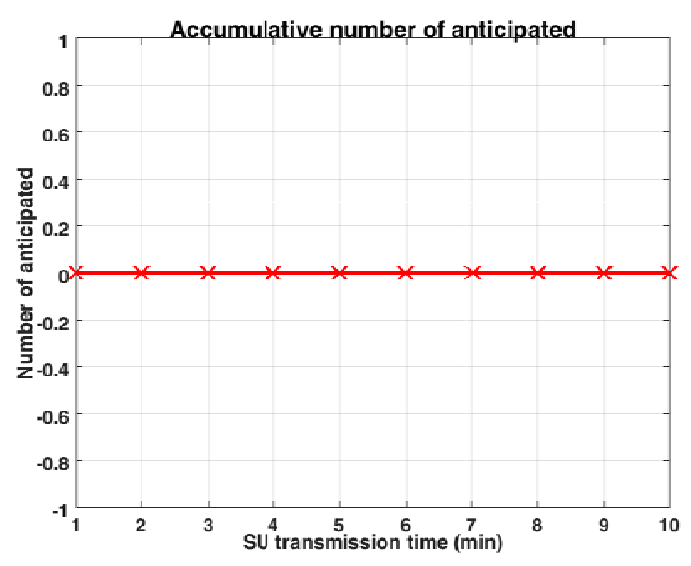

a) Number of anticipated (low Trank)

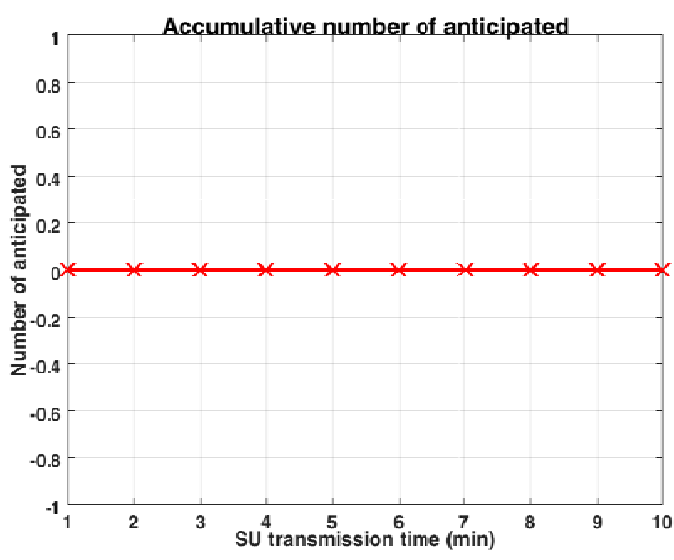

b) Number of antichated (Hileh Trainc)

Fig. 1. Anticipated Handoffs

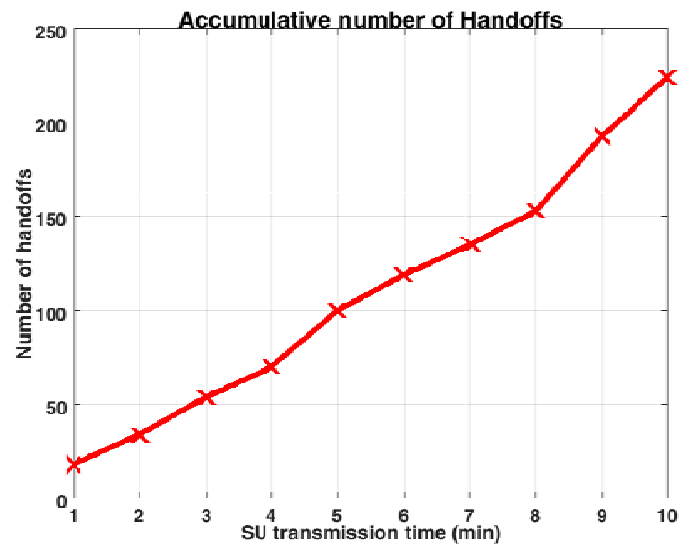

a) Mumber of bandofís flow Trafic)

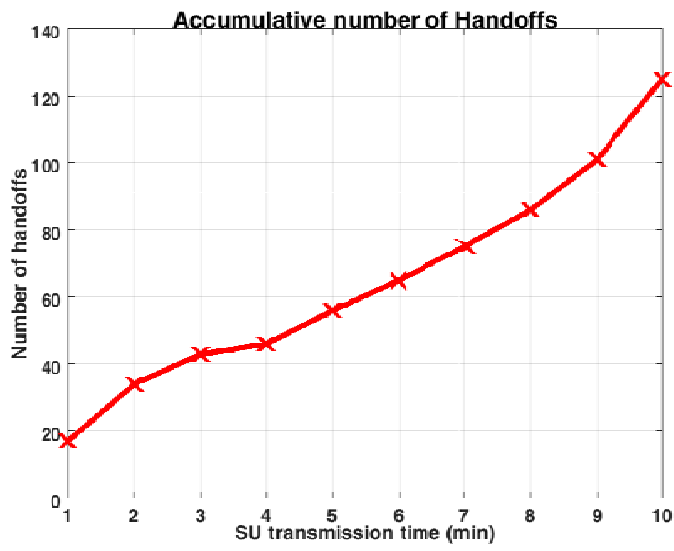

b) Number of handofls (High Traficd

Fig. 2. Number of Successful Handoffs

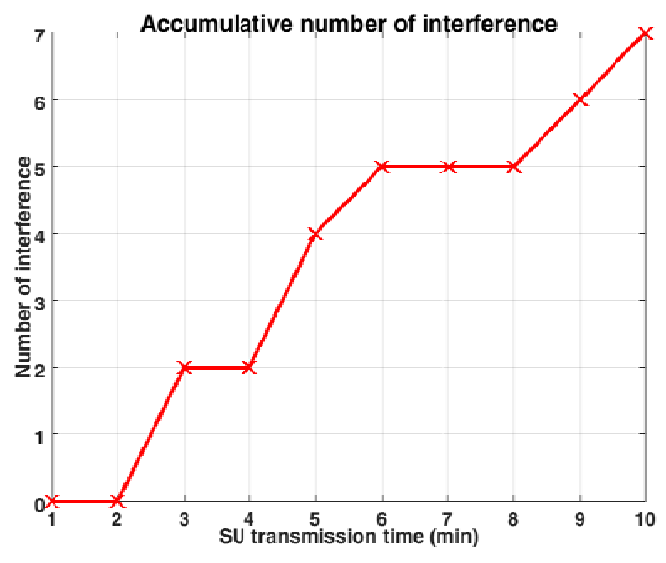

as Number of hoerference flow Trailich

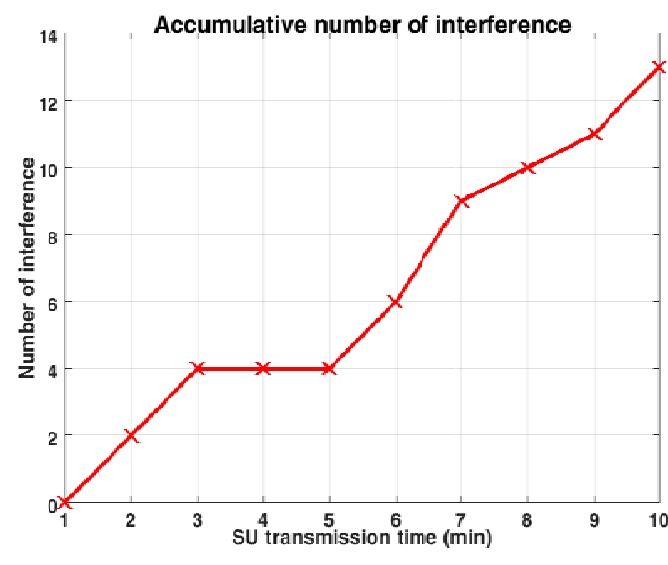

b) Number of merlerence (High Tranic)

Fig. 3. Number of Handoffs with Interference 

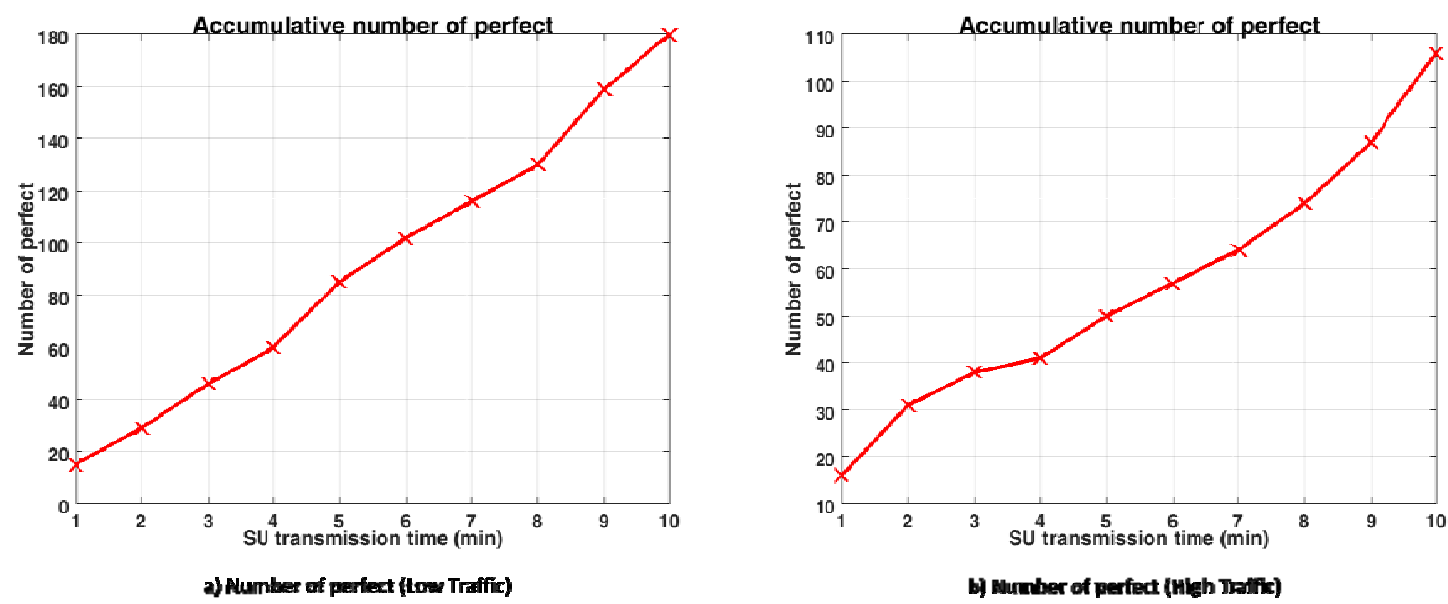

Fig. 4. Number of Perfect Handoffs
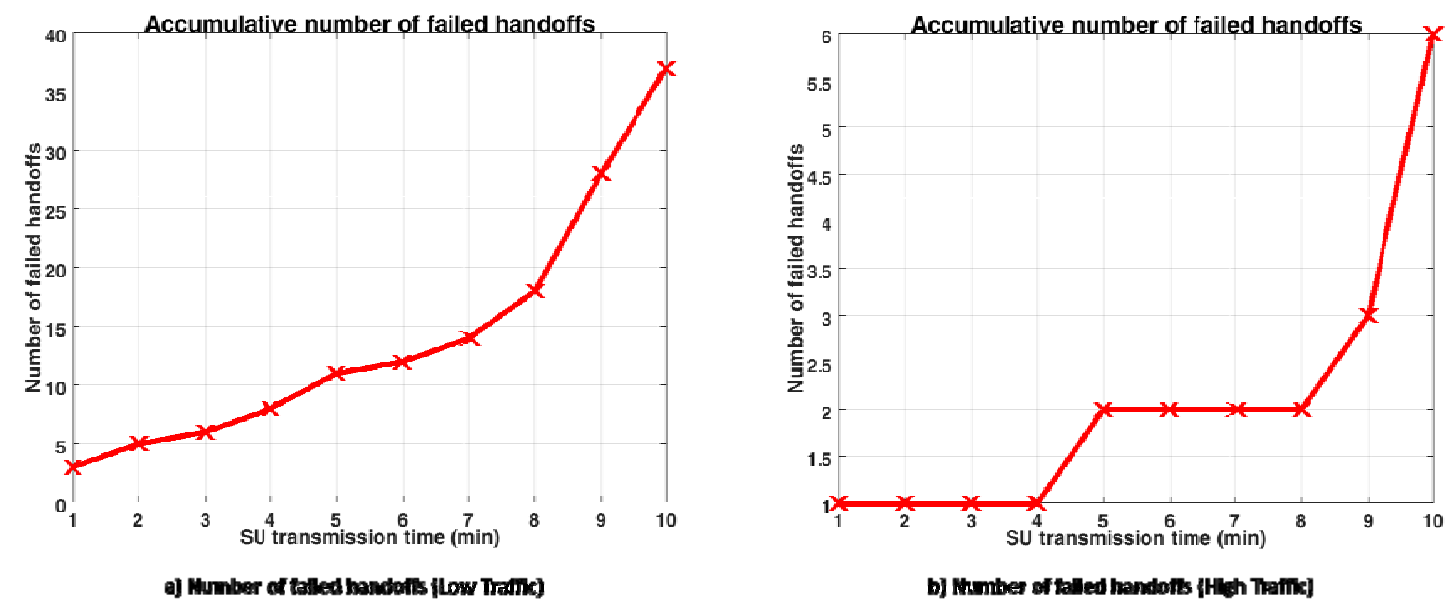

Fig. 5. Number of Failed Handoffs
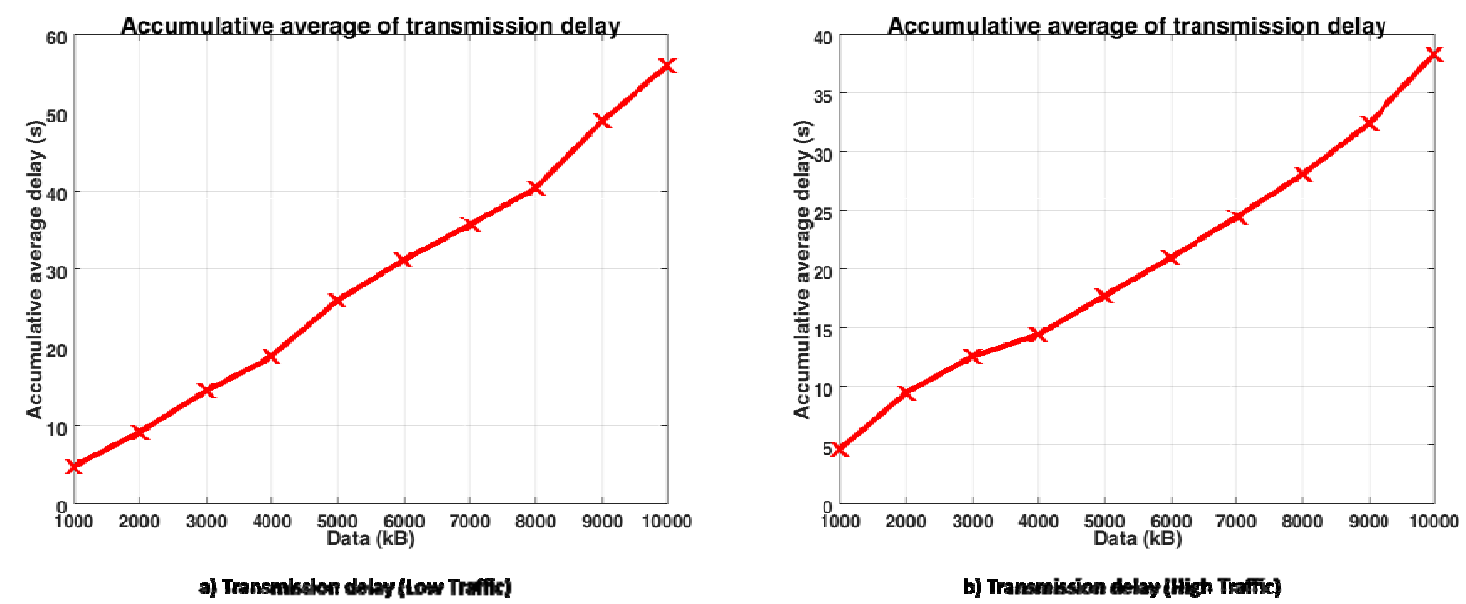

Fig. 6. Cumulative Average Delay

The bandwidth and throughput have a stable behavior in the two scenarios. However, the bandwidth assignment and throughput were higher in high traffic mainly because of a lower number of failed handoffs which led to lesser handoffs with interference and successful handoffs (see Fig. 9 and 10). 

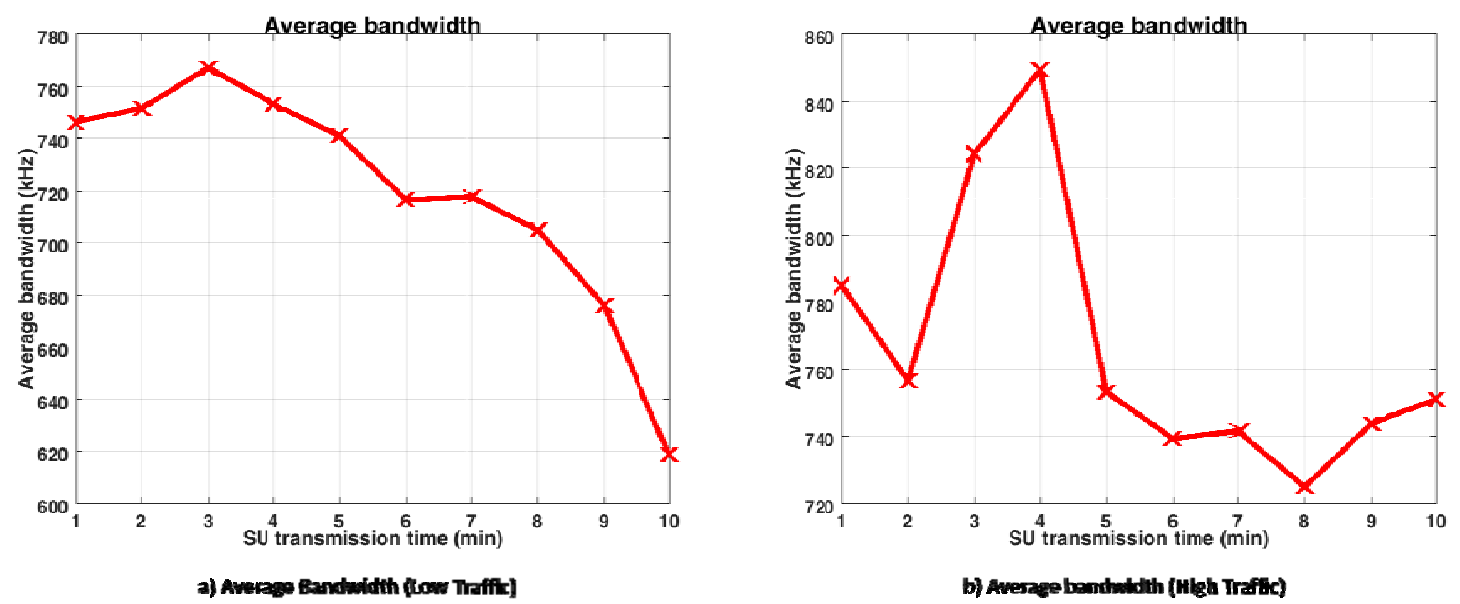

Fig. 7. Average Bandwidth
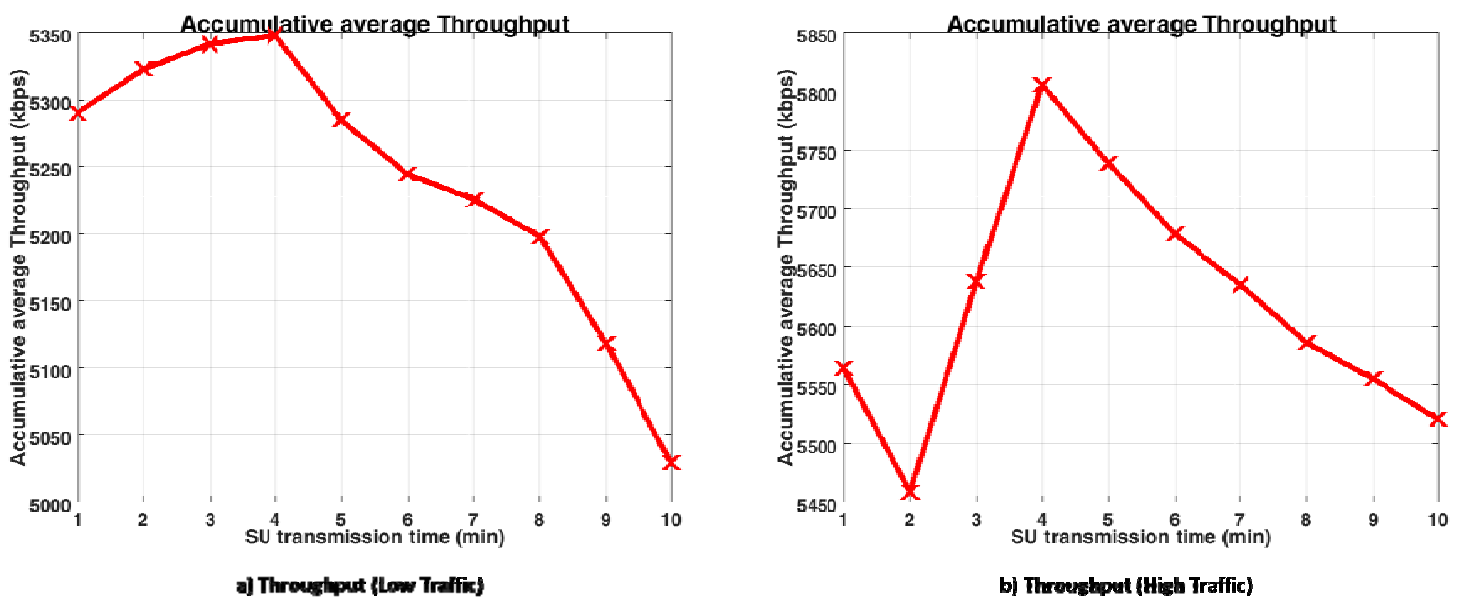

Fig. 8. Average Throughput

\section{Conclusions}

It is observed that the number of anticipated handoffs was zero in both scenarios indicating that the algorithm makes an efficient use of the anticipation policies based on the predictions carried out. Since it is an algorithm specialized in solving dichotomous problems, it positively affects the channel assignment policies.

The algorithm behaves in the optimal way for the high traffic scenario as the results showed which indicates that it is essential to handle a training base with more heterogeneous spectral occupancy data. This can be seen in high traffic scenarios due to the adjustment of the weights $\theta$ and a more effective prediction of the channel availability values.

\section{ACKNOWLEDGEMENTS}

The authors would like to thank to Colciencias and the Universidad Distrital Francisco José de Caldas for the support given during the research process of this project.

\section{REFERENCES}

[1] I. F. Akyildiz, W. Y. Lee, M. C. Vuran y S. Mohanty, «NeXt generation/dynamic spectrum access/cognitive radio wireless networks: A survey,» Computer networks, vol. 50, no 13, pp. 2127-2159, 2006.

[2] S. Haykin, «Cognitive radio: brain-empowered wireless communications,» IEEE journal on selected areas in communications, vol. 23, $\mathrm{n}^{\circ}$ 2, pp. 201-220, 2005.

[3] T. Yucek y H. Arslan, «A survey of spectrum sensing algorithms for cognitive radio applications,» IEEE communications surveys \& tutorials, vol. 11, no 1, pp. 116-130, 2009.

[4] E. Ahmed, A. Gani, S. Abolfazli, L. J. Yao y S. U. Khan, «Channel Assignment Algorithms in Cognitive Radio Networks: Taxonomy, Open Issues, and Challenges,» IEEE Commun. Surv. Tutorials, vol. 18, nº 1, pp. 795-823, 2016.

[5] G. Tsiropoulos, O. Dobre, M. Ahmed y K. Baddour, «Radio Resource Allocation Techniques for Efficient Spectrum Access in Cognitive Radio Networks,» IEEE Commun. Surv. Tutorials, vol. 18, nº 1, pp. 824-847, 2016.

[6] M. Ozger y O. B. Akan, «On the utilization of spectrum opportunity in cognitive radio networks,» IEEE Commun. Lett, vol. 20, $\mathrm{n}^{\circ} 1$, pp. 157-160, 2016

[7] Y. Liang, M. L. Reyes y J. D. Lee, «Real-Time Detection of Driver Cognitive Distraction,» IEEE transactions on intelligent transportation systems, vol. 8, $\mathrm{n}^{\circ} 2$, pp. 340-350, 2007. 
[8] H. I. Volos, C. I. Phelps y R. M. Buehrer, «Physical layer cognitive engine for multi-antenna systems,» de Military Communications Conference, 2008.

[9] S. Yarkan y H. Arslan, «Binary Time Series Approach to Spectrum Prediction for Cognitive Radio,» de Vehicular technology conference, 2007.

[10] A. Gorci, H. Celebi, K. A. Qaraqe y H. Arslan, «An autoregressive approach for spectrum occupancy modeling and prediction based on synchronous measurements,» de Personal Indoor and Mobile Radio Communications (PIMRC), 2011.

[11] N. Nagaraja, G. Kadambi y Y. Vershinin, «Co-operative spectrum sensing using CR node binary decision regression on estimated wireless path-loss curves,» de Electronics and Communication Systems (ICECS), 2014.

[12] S. Srivastava, M. Hashmi, S. Das y D. Barua, «Real-time blind spectrum sensing using USRP,» de Circuits and Systems (ISCAS), 2015.

\section{Author Profile}

Hans Raul Marquez Ramos Electronic Engineer, Master in Information Science and Communications. Young researcher and innovator of Colciencias and Universidad Distrital Francisco José de Caldas, Bogotá, Colombia.

Cesar Augusto Hernandez Suarez Electronic Engineer, Master in Information Science and Communications, Doctor of Philosophy in Systems and Computer Engineering of the Universidad Nacional de Colombia. Professor and researcher at Universidad Distrital Francisco José de Caldas, Bogotá, Colombia.

Diego Armando Giral Ramirez Electrical Engineer, Master in electric engineering. Professor at Universidad Distrital Francisco José de Caldas, Bogotá, Colombia. 